Description of Spreadsheet Calculations for Populating Data Tables of the Ecological Risk Assessment (Appendix B of the Human Health and Ecological Risk Assessment Document) for the Explosives Waste Treatment Facility (EWTF) at Site 300

J. Daniels

October 10, 2007 
This document was prepared as an account of work sponsored by an agency of the United States government. Neither the United States government nor Lawrence Livermore National Security, LLC, nor any of their employees makes any warranty, expressed or implied, or assumes any legal liability or responsibility for the accuracy, completeness, or usefulness of any information, apparatus, product, or process disclosed, or represents that its use would not infringe privately owned rights. Reference herein to any specific commercial product, process, or service by trade name, trademark, manufacturer, or otherwise does not necessarily constitute or imply its endorsement, recommendation, or favoring by the United States government or Lawrence Livermore National Security, LLC. The views and opinions of authors expressed herein do not necessarily state or reflect those of the United States government or Lawrence Livermore National Security, LLC, and shall not be used for advertising or product endorsement purposes.

This work performed under the auspices of the U.S. Department of Energy by Lawrence Livermore National Laboratory under Contract DE-AC52-07NA27344. 


\section{Description of Spreadsheet Calculations for Populating Data Tables of the Ecological Risk Assessment (Appendix B of the Human Health and Ecological Risk Assessment Document) for the Explosive Waste Treatment Facility (EWTF) at Site 300.}

This ecological risk assessment (ERA) is a supplement to the human health risk assessment (HRA) for the Explosive Waste Treatment Facility (EWTF). The EWTF is located near the center of Site 300 in a small, isolated canyon (see Figures 2 through 6 in the text). The ERA described in detail in Appendix B was prepared in accordance with guidance on currently accepted practice provided by the Human and Ecological Risk Division (HERD) at the Department of Toxic Substances Control (DSTC) of the State of California Environmental Protection Agency (CalEPA) in Sacramento, California.

The technical basis for this ERA is an analysis that involves a series of screening calculations to assess each of 21 contaminants of potential ecological concern (CPECs) for its potential to produce an adverse ecological impact in particular wildlife species, including vegetation, considered representative receptors of ecological interest (RREI) in the trophic levels of the food network at Site 300. This series of screening calculations is designed to illustrate whether CPECs identified as being of possible consequence in the most conservative screening calculation actually may be of lesser or no significance when more information is considered in subsequent screening calculations.

All of the series of screening calculations are based on a ratio between a soil concentration for a CPEC at a specific location $\left(\mathrm{mg}_{\mathrm{CPEC}} / \mathrm{kg}_{\text {soil }}\right)$ and a corresponding location-specific ecological soil screening level $\left(\mathrm{ESSL}_{\mathrm{LS}} ; \mathrm{mg}_{\mathrm{CPEC}} / \mathrm{kg}_{\text {soil }}\right)$. Such a ratio of concentration values for a CPEC is the location-specific ecological hazard quotient $\left(\mathrm{EHQ}_{\mathrm{LS}}\right)$ for that CPEC. Any $\mathrm{EHQ}_{\mathrm{LS}}$ that exceeds one indicates that the CPEC may be of possible consequence; however, the $\mathrm{ESSL}_{\mathrm{LS}}$ used as the denominator of the $\mathrm{EHQ}_{\mathrm{LS}}$ ratio may either be applicable to an individual RREI, or be a most conservative (lowest) value ESSL $_{\mathrm{LS}}$ selected from among all of the ESSL $_{\mathrm{LS}}$ values derived for each of the members of each RREI category (e.g., animal wildlife organisms, consisting of mammals, birds, reptiles, and invertebrates; or vegetation, consisting of all plants). In this latter case, the $\mathrm{EHQ}_{\mathrm{LS}}$ will be the most conservative one (i.e., the lowest ESSL $\mathrm{LS}_{\mathrm{S}}$ will appear as the denominator in each of the $\mathrm{EHQ}_{\mathrm{LS}}$ calculations). Specifically, the location-specific most conservative (lowest) minimum ecological soil screening level (ESSL $\mathrm{LS}_{\mathrm{Lmin}}$ ) value for a

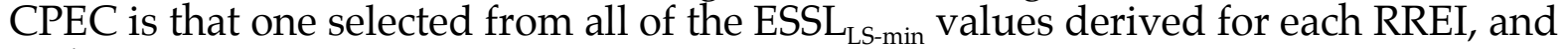
each individual ESSL ${ }_{\text {LS-min }}$ value for an RREI applicable to a particular CPEC is obtained using either the lowest toxic reference value $\left(\mathrm{TRV}_{\mathrm{Lo}}\right)$ available for that CPEC with respect to that RREI or an $\mathrm{ESSL}_{\mathrm{LS} \text {-min }}$ already available in the literature. In this case, using this most conservative (lowest) $\mathrm{ESSL}_{\mathrm{LS} \text {-min }}$ as the denominator of the $\mathrm{EHQ}_{\mathrm{LS}}$ equation for a CPEC will yield an $\mathrm{EHQ}_{\mathrm{LS} \text {-max }}$ value for that CPEC that is the most conservative for the category of RREIs (e.g., animal wildlife organisms). Thus, any CPEC with an $\mathrm{EHQ}_{\mathrm{LS} \text {-max }}>1$ suggests it may be of potential consequence to an RREI or the food web and so that CPEC deserves further assessment. As part of that further assessment, some additional calculations were also performed. 
The food network at Site 300 consists of nine different wildlife organisms plus vegetation, which represent a total of 10 individual RREIs across the different trophic levels. The nine RREIs composing wildlife organisms are one category of RREI and vegetation is another, due primarily to limitations in data with respect to deriving ESSL $_{\mathrm{LS}}$ values for CPECs for vegetation.

There are seven steps involved in performing the series of screening analyses that constitute this ERA analysis. These steps are described in Appendix B of the Human Health and Ecological Risk Assessment document for Site 300 (Gallegos et al., 2007, Volume 1). The calculations that produced the results for this ecological risk assessment that appear in Tables B-1 to B-23 were generated in four Microsoft Excel workbooks:

- ERACalcsforB-1toB-11;

- ERACalcsforB-12toB-16;

- ERACalcsforB-17min,B-18,B-20toB-21; and

- ERACalcsforB-17max,B-19,B-22toB-23.

Tables 1 through 4 in this document identify the locations in each Excel Workbook that yield results corresponding to information in Tables B-1 through B-23 of Appendix B. Accordingly, these tables, in combination with the four Excel workbooks mentioned make transparent the arithmetic involved in performing the calculations that were used for the ecological risk assessment applicable to the EWTF at Site 300. 
Table 1. ERACalcsforB-1toB-11; containing data appearing in Tables B-1 through B-11 of Appendix B, including ESSLLS-min; and derived EHQLS-max values for CPECs applicable to animal and plant wildlife.

\begin{tabular}{|c|c|c|c|}
\hline $\begin{array}{l}\text { Table in App. B of } \\
\text { EWTF Report } \\
\text { (Gallegos et al., 2007) }\end{array}$ & Worksheet name & \multicolumn{2}{|c|}{$\begin{array}{l}\text { Columns in worksheet with data that } \\
\text { appear in specified table of App. B }\end{array}$} \\
\hline \multirow{3}{*}{$\begin{array}{l}\text { Table B-1 } \\
\text { (pp. B-24 \& B-25) }\end{array}$} & ChemINPUT & \multicolumn{2}{|l|}{ Cols. C, A, J } \\
\hline & MammalINPUT & \multicolumn{2}{|l|}{ Cols. C, A, E, F, G } \\
\hline & AVIAN_REPTILEINPUT & \multicolumn{2}{|l|}{ Cols. C, A, E, F, G } \\
\hline $\begin{array}{l}\text { Table B-2 } \\
\text { (p. B-26) }\end{array}$ & EcoReceptors & \multicolumn{2}{|c|}{ Cols. B, C, D, E, F, G, H, I, J, K } \\
\hline \multirow{3}{*}{$\begin{array}{l}\text { Table B-3 } \\
\text { (p. B-27) }\end{array}$} & ChemINPUT & \multicolumn{2}{|l|}{ Cols. C, A } \\
\hline & MammalINPUT & \multicolumn{2}{|l|}{ Cols. C, A, D, H } \\
\hline & AVIAN_REPTILEINPUT & \multicolumn{2}{|l|}{ Cols. C, A, D, H } \\
\hline \multirow{7}{*}{$\begin{array}{l}\text { Table B-4 } \\
\text { (pp. B-28 \& B-29) }\end{array}$} & ChemINPUT & \multicolumn{2}{|l|}{ Cols. C } \\
\hline & $\begin{array}{l}\text { EWTF } \\
\text { MammalRREI }\end{array}$ & \multicolumn{2}{|c|}{ Cols. C, D, E, F, G, H, I } \\
\hline & $\begin{array}{l}\text { RANCH } \\
\text { MammalRREI }\end{array}$ & \multicolumn{2}{|c|}{ Cols. C, D, E, F, G, H, I } \\
\hline & $\begin{array}{l}\text { OtherLocs } \\
\text { MammalRREI }\end{array}$ & \multicolumn{2}{|c|}{ Cols. C, D, E, F, G, H, I } \\
\hline & EWTF AVIAN_REPTILERREI & \multicolumn{2}{|l|}{ Cols. C, D, E, F } \\
\hline & $\begin{array}{l}\text { RANCH } \\
\text { AVIAN_REPTILERREI }\end{array}$ & \multicolumn{2}{|l|}{ Cols. C, D, E, F } \\
\hline & $\begin{array}{l}\text { OtherLocs } \\
\text { AVIAN_REPTILERREI }\end{array}$ & \multicolumn{2}{|l|}{ Cols. C, D, E, F } \\
\hline $\begin{array}{l}\text { Table B-5a: } \\
\text { BAF regression } \\
\text { coefficients and median } \\
\text { values } \\
\text { (p. } 30)\end{array}$ & BAF data & \multicolumn{2}{|c|}{ Cols. A through J (as applicable) } \\
\hline $\begin{array}{l}\text { Table B-5b: } \\
\text { Location-specific BAF } \\
\text { values for vegetation, } \\
\text { invertebrates, and small } \\
\text { mammals } \\
\text { (pp. B-31 \& B-32) }\end{array}$ & $\begin{array}{ll}\text { ChemINPUT: } & \text { EWTF } \\
& \text { B812 } \\
& \text { B895 } \\
& \text { East Pasture } \\
& \text { Carnegie } \\
& \text { Ranch }\end{array}$ & $\begin{array}{ll}\text { Cols. } & \mathrm{K}, \mathrm{L}, \mathrm{M} \\
\text { Cols. } & \mathrm{N}, \mathrm{O}, \mathrm{P} \\
\text { Cols. } & \mathrm{Q}, \mathrm{R}, \mathrm{S} \\
\text { Cols. } & \mathrm{T}, \mathrm{U}, \mathrm{V} \\
\text { Cols. } & \mathrm{W}, \mathrm{X}, \mathrm{Y} \\
\text { Cols. } & \mathrm{Z}, \mathrm{AA}, \mathrm{AB}\end{array}$ & $\begin{array}{l}\text { (veg, INV, Sm. Mam) } \\
\text { (veg, INV, Sm. Mam) } \\
\text { (veg, INV, Sm. Mam) } \\
\text { (veg, INV, Sm. Mam) } \\
\text { (veg, INV, Sm. Mam) } \\
\text { (veg, INV, Sm. Mam) }\end{array}$ \\
\hline
\end{tabular}


Table 1. (continued)

\begin{tabular}{|c|c|c|}
\hline $\begin{array}{l}\text { Table in App. B of } \\
\text { EWTF Report } \\
\text { (Gallegos et al., 2007) }\end{array}$ & Worksheet name & $\begin{array}{l}\text { Columns in worksheet with data that } \\
\text { appear in specified table of App. B }\end{array}$ \\
\hline \multirow{6}{*}{$\begin{array}{l}\text { Table B-6a: } \\
\text { Organism specific } \\
\text { ESSLLS-min for EWTF } \\
\text { location } \\
\text { (p. B-33) }\end{array}$} & ChemINPUT & Cols. C \\
\hline & MammalINPUT & Col. $\mathrm{H}$ \\
\hline & AVIAN_REPTILEINPUT & Col. $\mathrm{H}$ \\
\hline & $\begin{array}{l}\text { EWTF } \\
\text { MammalRREI }\end{array}$ & Cols. C, J, K, L, M, N, O \\
\hline & EWTF AVIAN_REPTILERREI & Cols. C, G, H, I \\
\hline & INVERTessI & Cols. C, D \\
\hline \multirow{6}{*}{$\begin{array}{l}\text { Table B-6b: } \\
\text { Organism specific } \\
\text { ESSL LS-min for Connolly } \\
\text { Ranch location } \\
\text { (p. B-34) }\end{array}$} & ChemINPUT & Cols. C \\
\hline & MammalINPUT & Col. H \\
\hline & AVIAN_REPTILEINPUT & Col. H \\
\hline & $\begin{array}{l}\text { Ranch } \\
\text { MammalRREI }\end{array}$ & Cols. C, J, K, L, M, N, O \\
\hline & Ranch AVIAN_REPTILERREI & Cols. C, G, H, I \\
\hline & INVERTessI & Cols. C, D \\
\hline $\begin{array}{l}\text { Table B-7: } \\
\text { ESSL LS-min for all } \\
\text { locations } \\
\text { (p. B-35) }\end{array}$ & AllLoctnsEHQs & 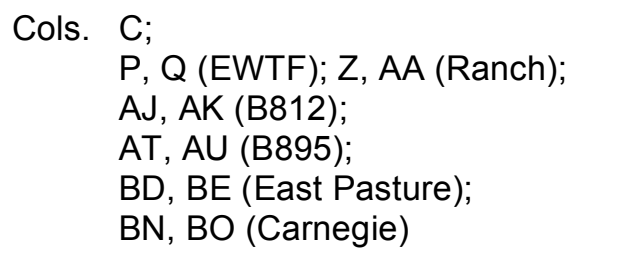 \\
\hline \multirow{3}{*}{$\begin{array}{l}\text { Table B-8: } \\
\text { Modeled concentrations } \\
\text { for chemicals of concern } \\
\text { at all } 6 \text { locations } \\
\text { (p. B-36) }\end{array}$} & ChemINPUT & Cols. H, I, E, F, G, D \\
\hline & ALLLocatnsEHQ & Cols. H, I, E, F, G, D \\
\hline & PLANTSonly & Cols. H, I, E, F, G, D \\
\hline $\begin{array}{l}\text { Table B-9: } \\
\text { EHQLS-max based on } \\
\text { most conservative } \\
\text { (lowest) ESSL } \\
6 \text { locations } \\
\text { (p. B-37) }\end{array}$ & ALLLocatnsEHQ & $\begin{array}{ll}\text { Cols. } & \text { C; } \\
& \text { R (EWTF); AB (Ranch); } \\
\text { AL (B812); } \\
\text { AV (B895); } \\
\text { BF (East Pasture); } \\
\text { BP (Carnegie) }\end{array}$ \\
\hline
\end{tabular}


Table 1. (continued)

\begin{tabular}{|c|c|c|c|}
\hline $\begin{array}{l}\text { Table in App. B of } \\
\text { EWTF Report } \\
\text { (Gallegos et al., 2007) }\end{array}$ & Worksheet name & $\begin{array}{l}\text { Colur } \\
\text { appe }\end{array}$ & $\begin{array}{l}\text { ns in worksheet with data that } \\
\text { in specified table of App. B }\end{array}$ \\
\hline \multirow{4}{*}{$\begin{array}{l}\text { Table B-10a: } \\
\text { Kit Fox ESSL } L_{\llcorner S-m i n} \text { and } \\
\text { EHQLS-max at all } 6 \\
\text { locations } \\
\text { (pp. B-38 \& B-39) }\end{array}$} & Chemlnput & Cols. & $\mathrm{D}, \mathrm{E}, \mathrm{F}, \mathrm{G}, \mathrm{H}, \mathrm{I}$ \\
\hline & $\begin{array}{l}\text { EWTF } \\
\text { MammalRREI }\end{array}$ & Cols. & $\begin{array}{l}\text { S (Modeled soil concentration) } \\
\text { N (Kit Fox ESSLLS-min) } \\
\text { T (EHQLS-max @ EWTF) }\end{array}$ \\
\hline & $\begin{array}{l}\text { Ranch } \\
\text { MammalRREI }\end{array}$ & Cols. & $\begin{array}{l}\text { S (Modeled soil concentration) } \\
\text { N (Kit Fox ESSLLS-min) } \\
\text { T (EHQLS-max @ Ranch) }\end{array}$ \\
\hline & $\begin{array}{l}\text { OtherLoc } \\
\text { MammalRREI }\end{array}$ & Cols. & $\begin{array}{l}\text { S (Modeled soil concentration) } \\
\text { N (Kit Fox ESSL LS-min) } \\
\text { T (EHQLS-max @ "location") } \\
\text { W (Cumulative EHQLS-max) }\end{array}$ \\
\hline \multirow{4}{*}{$\begin{array}{l}\text { Table B-10b: } \\
\text { Burrowing owl ESSL } L_{L S-} \\
\min \text { and } E H Q_{L S-\max } \text { at all } 6 \\
\text { locations } \\
\text { (pp. B-40 \& B-41) }\end{array}$} & Chemlnput & Col. & $D, E, F, G, H, I$ \\
\hline & EWTF AVIAN_REPTILERREI & Cols. & $\begin{array}{l}\text { M (Modeled soil concentration) } \\
\mathrm{H} \text { (Burrowing Owl ESSL LS-min) } \\
\mathrm{N}(\text { (EHQLS-max @ EWTF) }\end{array}$ \\
\hline & $\begin{array}{l}\text { RANCH } \\
\text { AVIAN_REPTILERREI }\end{array}$ & Cols. & $\begin{array}{l}\text { M (Modeled soil concentration) } \\
\text { H (Burrowing Owl ESSL LS-min) } \\
\text { N (EHQLS-max @ Ranch) }\end{array}$ \\
\hline & $\begin{array}{l}\text { OtherLocs } \\
\text { AVIAN_REPTILERREI }\end{array}$ & Cols. & $\begin{array}{l}\text { M (Modeled soil concentration) } \\
\text { H (Burrowing Owl ESSL LS-min) } \\
\text { N (EHQLS-max @ "location") }\end{array}$ \\
\hline \multirow{2}{*}{$\begin{array}{l}\text { Table B-11: } \\
\text { PLANTS-Comparing } \\
\text { EHQLS-min for } \\
\text { MEASURED and } \\
\text { MODELED soil } \\
\text { concentrations } \\
\text { (p. B-42) }\end{array}$} & Chemlnput & Cols. & $C, D, E, F, G, H, I$ \\
\hline & PLANTSonly & Cols. & $\begin{array}{l}\text { D-I (modeled soil concentration); } \\
\text { N (measured soil concentration); } \\
\text { J, K, L, M (ESSL } \text { LS-min }) ; \\
\text { O (EHQ } \text { measured-max); } \\
\text { D, P (EWTF); } \\
\text { E, Q (B812); } \\
\text { F, R (B895); } \\
\text { G, S (East Pasture); } \\
\text { H, T (Carnegie); } \\
\text { I, U (Ranch) } \\
\text { P-U (modeled to measured ratios) }\end{array}$ \\
\hline
\end{tabular}


Table 2. ERACalcsforB-12toB-16; containing data appearing in Tables B-12 through B16 of Appendix B, including ESSL LS-max; ; and derived EHQLS-min values for CPECs applicable to vertebrate wildlife for which the $\mathrm{EHQ}_{\mathrm{LS}-\max }>1$ in Table B-9.

\begin{tabular}{|c|c|c|c|c|}
\hline $\begin{array}{l}\text { Table in App. B of } \\
\text { EWTF Report } \\
\text { (Gallegos et al., 2007) }\end{array}$ & \multicolumn{2}{|c|}{ Worksheet name } & \multicolumn{2}{|c|}{$\begin{array}{l}\text { Columns in worksheet with data that } \\
\text { appear in specified table of App. B }\end{array}$} \\
\hline \multirow{3}{*}{$\begin{array}{l}\text { Table B-12 } \\
\text { Experimental test } \\
\text { species (ETS) data } \\
\text { including TRV }{ }_{\text {ETS-Hi }} \\
\text { values } \\
\text { (p. B-43) }\end{array}$} & \multicolumn{2}{|l|}{ ChemINPUT } & \multicolumn{2}{|l|}{ Cols. C, A, J } \\
\hline & \multicolumn{2}{|c|}{ MammalINPUT } & \multicolumn{2}{|l|}{ Cols. C, A, E, F, G } \\
\hline & \multicolumn{2}{|c|}{ AVIAN_REPTILEINPUT } & \multicolumn{2}{|l|}{ Cols. C, A, E, F, G } \\
\hline \multirow{8}{*}{$\begin{array}{l}\text { Table B-13a: } \\
\text { TRV }_{\text {Hi values (including }} \text { valu, } \\
\text { those for Al, Cd, Cu, Pb, } \\
\text { and } \mathrm{Zn} \text { ) applicable to } \\
\text { vertebrate wildlife } \\
\text { RREls } \\
\text { (p. B-44) }\end{array}$} & \multicolumn{2}{|c|}{ MammalINPUT } & \multicolumn{2}{|l|}{ Col. G, H } \\
\hline & \multicolumn{2}{|c|}{ AVIAN_REPTILEINPUT } & \multicolumn{2}{|l|}{ Col. G, H } \\
\hline & \multicolumn{2}{|c|}{$\begin{array}{l}\text { EWTF } \\
\text { MammalRREI }\end{array}$} & \multicolumn{2}{|c|}{ Cols. C, D, E, F, G, H, I } \\
\hline & \multicolumn{2}{|c|}{$\begin{array}{l}\text { Ranch } \\
\text { MammalRREI }\end{array}$} & \multicolumn{2}{|c|}{ Cols. C, D, E, F, G, H, I } \\
\hline & \multicolumn{2}{|c|}{$\begin{array}{l}\text { OtherLoc } \\
\text { MammalRREI }\end{array}$} & \multicolumn{2}{|c|}{ Cols. C, D, E, F, G, H, I } \\
\hline & \multicolumn{2}{|c|}{$\begin{array}{l}\text { EWTF } \\
\text { AVIAN_ReptileRREI }\end{array}$} & \multicolumn{2}{|l|}{ Cols. C, D, E, F } \\
\hline & \multicolumn{2}{|c|}{$\begin{array}{l}\text { Ranch } \\
\text { AVIAN_ReptileRREI }\end{array}$} & \multicolumn{2}{|l|}{ Cols. C, D, E, F } \\
\hline & \multicolumn{2}{|c|}{$\begin{array}{l}\text { OtherLoc } \\
\text { AVIAN_ReptileRREI }\end{array}$} & \multicolumn{2}{|l|}{ Cols. C, D, E, F } \\
\hline \multirow[t]{7}{*}{$\begin{array}{l}\text { Table B-13b: } \\
\text { ESSL } \\
\text { (p. B-45) }\end{array}$} & ChemINPUT & $\begin{array}{l}\text { EWTF } \\
\text { B812 } \\
\text { B895 } \\
\text { East Pasture } \\
\text { Carnegie } \\
\text { Ranch }\end{array}$ & $\begin{array}{ll}\text { Cols. } & \mathrm{K}, \mathrm{L}, \mathrm{M} \\
\text { Cols. } & \mathrm{N}, \mathrm{O}, \mathrm{P} \\
\text { Cols. } & \mathrm{Q}, \mathrm{R}, \mathrm{S} \\
\text { Cols. } & \mathrm{T}, \mathrm{U}, \mathrm{V} \\
\text { Cols. } & \mathrm{W}, \mathrm{X}, \mathrm{Y} \\
\text { Cols. } & \mathrm{Z}, \mathrm{AA}, \mathrm{AB}\end{array}$ & $\begin{array}{l}\text { (veg, INV, Sm. Mam) } \\
\text { (veg, INV, Sm. Mam) } \\
\text { (veg, INV, Sm. Mam) } \\
\text { (veg, INV, Sm. Mam) } \\
\text { (veg, INV, Sm. Mam) } \\
\text { (veg, INV, Sm. Mam) }\end{array}$ \\
\hline & \multicolumn{2}{|l|}{ BAF data } & \multicolumn{2}{|c|}{ Cols. A through J (as applicable) } \\
\hline & \multicolumn{2}{|c|}{ EcoReceptors } & \multicolumn{2}{|c|}{ Cols. B, C, D, E, F, G, H, I, J, K } \\
\hline & \multicolumn{2}{|c|}{$\begin{array}{l}\text { EWTF } \\
\text { MammaIRREI }\end{array}$} & \multicolumn{2}{|c|}{ Cols. J, K, L, M, N, O } \\
\hline & \multicolumn{2}{|c|}{$\begin{array}{l}\text { Ranch } \\
\text { MammaIRREI }\end{array}$} & \multicolumn{2}{|c|}{ Cols. J, K, L, M, N, O } \\
\hline & \multicolumn{2}{|c|}{$\begin{array}{l}\text { OtherLoc } \\
\text { MammalRREI }\end{array}$} & \multicolumn{2}{|c|}{ Cols. J, K, L, M, N, O } \\
\hline & $\begin{array}{l}\text { EWTF } \\
\text { AVIAN_Rept }\end{array}$ & RREI & Cols. G, H, I & \\
\hline
\end{tabular}




\begin{tabular}{|c|c|c|}
\hline $\begin{array}{l}\text { Table in App. B of } \\
\text { EWTF Report } \\
\text { (Gallegos et al., 2007) }\end{array}$ & Worksheet name & $\begin{array}{l}\text { Columns in worksheet with data that } \\
\text { appear in specified table of App. B }\end{array}$ \\
\hline \multirow{3}{*}{$\begin{array}{l}\text { Table B-13b (cont.): } \\
\text { ESSL }_{\text {LS-max values }} \\
\text { (p. B-45) }\end{array}$} & $\begin{array}{l}\text { Ranch } \\
\text { AVIAN_ReptileRREI }\end{array}$ & Cols. G, H, I \\
\hline & $\begin{array}{l}\text { OtherLoc } \\
\text { AVIAN_ReptileRREI }\end{array}$ & Cols. G, H, I \\
\hline & INVERTessI & Cols. C, D \\
\hline $\begin{array}{l}\text { Table B-14: } \\
\text { RREI EHQLS-max } \\
\text { (p. B-46) }\end{array}$ & ALLLocsEHQs & $\begin{array}{l}\text { Cols. C, D; J-O (EWTF); } \\
\text { Cols. C, I; T-Y (Ranch); } \\
\text { Cols. C, E; AJ-Al (B812); } \\
\text { Cols. C, F; AN-AS (B895) } \\
\text { Cols. C, G; AX-BC (EstPstr) } \\
\text { Cols. C, H; BH-BM (Crngie) }\end{array}$ \\
\hline $\begin{array}{l}\text { Table B-15: } \\
\text { Most conservative } \\
\text { (lowest)EHQLS-max } \\
\text { (p. B-47) }\end{array}$ & ALLLocsEHQs & $\begin{array}{l}\text { Cols. [C, D; J-O]; P, Q, R (EWTF); } \\
\text { Cols. [C, I; T-Y]; Z, AA, AB (Ranch); } \\
\text { Cols. [C, E; AJ-AI]; AJ, AK, AL (B812); } \\
\text { Cols. [C, F; AN-AS]; AT, AU, AV (B895) } \\
\text { Cols. [C, G; AX-BC]; BD, BE, BF (EstPstr) } \\
\text { Cols. [C, H; BH-BM]; BN, BO, BP (Crngie) }\end{array}$ \\
\hline \multirow{3}{*}{$\begin{array}{l}\text { Table B-16a: } \\
\text { Kit Fox ESSL } L_{L S-m i n} \text { and } \\
\text { EHQLS-max } \\
\text { (p. B-48) }\end{array}$} & $\begin{array}{l}\text { EWTF } \\
\text { MammalRREI }\end{array}$ & Cols. S, N T \\
\hline & $\begin{array}{l}\text { Ranch } \\
\text { MammalRREI }\end{array}$ & Cols. S, N, T \\
\hline & $\begin{array}{l}\text { OtherLoc } \\
\text { MammaIRREI }\end{array}$ & Cols. S, N, T \\
\hline \multirow{3}{*}{$\begin{array}{l}\text { Table B-16b: } \\
\text { Burrowing Owl } \\
\text { ESSL LS-min and } \\
\text { EHQLS-max } \\
\text { (p. B-49) }\end{array}$} & $\begin{array}{l}\text { EWTF } \\
\text { AVIAN_ReptileRREI }\end{array}$ & Cols. M, H, N \\
\hline & $\begin{array}{l}\text { Ranch } \\
\text { AVIAN_ReptileRREI }\end{array}$ & Cols. M, H, N \\
\hline & $\begin{array}{l}\text { OtherLoc } \\
\text { AVIAN_ReptileRREI }\end{array}$ & Cols. M, H, N \\
\hline
\end{tabular}


Table 3. ERACalcsforB-17min, B-18, B-20 to B-21; containing data appearing in Tables B-17a; 17b for ESSLLS-min, B-18 [EHQLS-max], B-20 through B-21 of Appendix B for measured concentrations of metals.

\begin{tabular}{|c|c|c|}
\hline $\begin{array}{l}\text { Table in App. B of } \\
\text { EWTF Report } \\
\text { (Gallegos et al., 2007) }\end{array}$ & Worksheet name & $\begin{array}{l}\text { Columns in worksheet with data that appear } \\
\text { in specified table of App. B }\end{array}$ \\
\hline \multirow[t]{2}{*}{$\begin{array}{l}\text { Table B-17a: } \\
\text { Site } 300 \text { Measured } \\
\text { concentrations and } \\
\text { BAFs } \\
\text { (p. B-50) }\end{array}$} & ChemINPUT & $\begin{array}{llr}\text { Cols. C; } & & \\
\text { Cols. } & \text { K, L, M(veg, INV, Sm. Mam) } \\
\text { Cols. } & \text { N, O, P } & \text { (veg, INV, Sm. Mam) } \\
\text { Cols. } & \text { Q, R, S } & \text { (veg, INV, Sm. Mam) } \\
\text { Cols. } & \text { T, U, V (veg, INV, Sm. Mam) } \\
\text { Cols. } & \text { W, X, Y } & \text { (veg, INV, Sm. Mam) } \\
\text { Cols. } & \text { Z, AA, AB } & \text { (veg, INV, Sm. Mam) }\end{array}$ \\
\hline & BAF data & Cols. A through J (as applicable) \\
\hline \multirow{7}{*}{$\begin{array}{l}\text { Table B-17b: } \\
\text { Site 300; RREI specific } \\
\text { ESSL } \\
\text { (p. B-51) }\end{array}$} & $\begin{array}{l}\text { S300EWTF } \\
\text { MammaIRREI }\end{array}$ & Cols. J, K, L, M, N, O \\
\hline & $\begin{array}{l}\text { S300Ranch } \\
\text { MammalRREI }\end{array}$ & Cols. J, K, L, M, N, O \\
\hline & $\begin{array}{l}\text { S300OtherLoc } \\
\text { MammalRREI }\end{array}$ & Cols. J, K, L, M, N, O \\
\hline & $\begin{array}{l}\text { S300EWTF } \\
\text { AVIAN_ReptileRREI }\end{array}$ & Cols. G, H, I \\
\hline & $\begin{array}{l}\text { S300Ranch } \\
\text { AVIAN_ReptileRREI }\end{array}$ & Cols. G, H, I \\
\hline & $\begin{array}{l}\text { S300OtherLoc } \\
\text { AVIAN_ReptileRREI }\end{array}$ & Cols. G, H, I \\
\hline & INVERTessI & Cols. C; D \\
\hline $\begin{array}{l}\text { Table B-18: } \\
\text { Comparing EHQLS-max } \\
\text { for Site } 300 \\
\text { MEASURED and } \\
\text { location MODELED soil } \\
\text { concentrations with } \\
\text { respect to mammals } \\
\text { and avian species and } \\
\text { contributions } \\
\text { (p. B-52) }\end{array}$ & $\begin{array}{l}\text { S300ALLLocatns } \\
\text { EHQs\&Ratios }\end{array}$ & 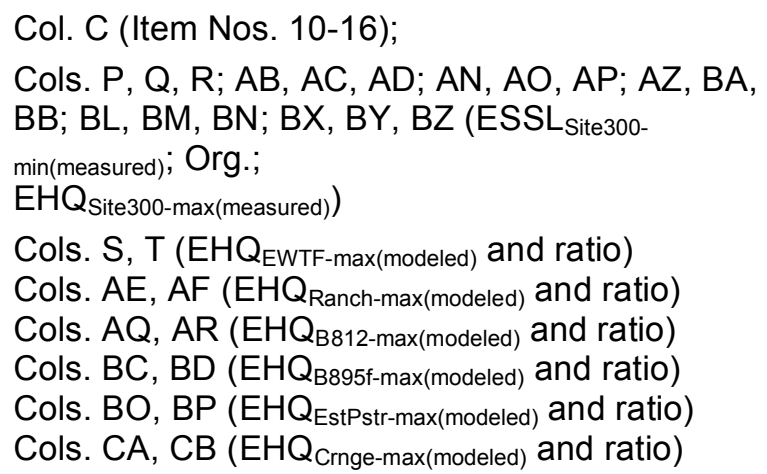 \\
\hline
\end{tabular}


Table 3. (continued)

\begin{tabular}{|c|c|c|}
\hline $\begin{array}{l}\text { Table in App. B of } \\
\text { EWTF Report } \\
\text { (Gallegos et al., 2007) }\end{array}$ & Worksheet name & $\begin{array}{l}\text { Columns in worksheet with data that appear } \\
\text { in specified table of App. B }\end{array}$ \\
\hline $\begin{array}{l}\text { Table B-20: } \\
\text { ESSL } \\
\text { EHS-min and } \\
\text { based on Sor Kite } 300 \\
\text { measured soil } \\
\text { concentrations } \\
\text { (p. B-54) }\end{array}$ & $\begin{array}{l}\text { S300EWTF } \\
\text { MammalRREI } \\
\text { S300Ranch } \\
\text { MammalRREI } \\
\text { S300OtherLoc } \\
\text { MammalRREI }\end{array}$ & $\begin{array}{l}\text { Cols. C, S, N, T } \\
\text { Cols. C, S, N, T } \\
\text { Cols. C, S, N, T and W }\end{array}$ \\
\hline $\begin{array}{l}\text { Table B-21: } \\
\text { ESSL } L_{L S-m i n} \text { and } E_{\text {HQS- }} \\
\max \text { for Burrowing Owl } \\
\text { based on Site } 300 \\
\text { measured soil } \\
\text { concentrations } \\
\text { (p. B-54) }\end{array}$ & $\begin{array}{l}\text { S300EWTF } \\
\text { AVIAN_ReptileRREI } \\
\text { S300Ranch } \\
\text { AVIAN_ReptileRREI } \\
\text { S300OtherLoc } \\
\text { AVIAN_ReptileRREI }\end{array}$ & $\begin{array}{l}\text { Cols. C, M, H, N } \\
\text { Cols. C, M, H, N } \\
\text { Cols. C, M, H, N and Q }\end{array}$ \\
\hline
\end{tabular}


Table 4. ERACalcsforB-17max, B-19, B-22 to B-23; containing data appearing in Tables B-17b for ESSLLS-max, B-19 [EHQLS-min], B-22 through

B-23 of Appendix B for measured concentrations of metals.

\begin{tabular}{|c|c|c|}
\hline $\begin{array}{l}\text { Table in App. B of } \\
\text { EWTF Report } \\
\text { (Gallegos et al., 2007) }\end{array}$ & Worksheet name & $\begin{array}{l}\text { Columns in worksheet with data that appear } \\
\text { in specified table of App. B }\end{array}$ \\
\hline \multirow{7}{*}{$\begin{array}{l}\text { Table B-17b: } \\
\text { Site 300; RREI specific } \\
\text { ESSLLS-max } \\
\text { (p. B-51) }\end{array}$} & $\begin{array}{l}\text { S300EWTF } \\
\text { MammalRREI }\end{array}$ & Cols. J, K, L, M, N, O \\
\hline & $\begin{array}{l}\text { S300Ranch } \\
\text { MammalRREI }\end{array}$ & Cols. J, K, L, M, N, O \\
\hline & $\begin{array}{l}\text { S300OtherLoc } \\
\text { MammalRREI }\end{array}$ & Cols. J, K, L, M, N, O \\
\hline & $\begin{array}{l}\text { S300EWTF } \\
\text { AVIAN_ReptileRREI }\end{array}$ & Cols. G, H, I \\
\hline & $\begin{array}{l}\text { S300Ranch } \\
\text { AVIAN_ReptileRREI }\end{array}$ & Cols. G, H, I \\
\hline & $\begin{array}{l}\text { S300OtherLoc } \\
\text { AVIAN_ReptileRREI }\end{array}$ & Cols. G, H, I \\
\hline & INVERTessI & Cols. C; D \\
\hline $\begin{array}{l}\text { Table B-19: } \\
\text { Comparing EHQLS-min for } \\
\text { Site } 300 \text { MEASURED } \\
\text { and location MODELED } \\
\text { soil concentrations with } \\
\text { respect to mammals } \\
\text { and avian species and } \\
\text { contributions } \\
\text { (p. B-53) }\end{array}$ & $\begin{array}{l}\text { S300ALLLocatns } \\
\text { EHQs\&Ratios }\end{array}$ & 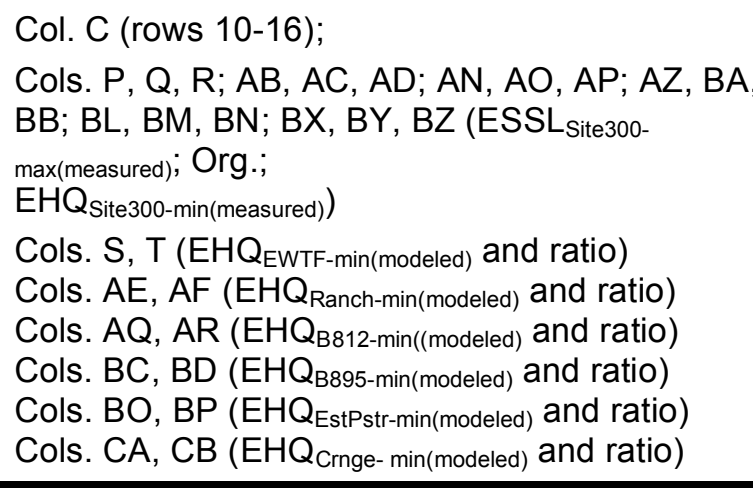 \\
\hline $\begin{array}{l}\text { Table B-22: } \\
\text { ESSL } \\
\text { EHQ-max and } \\
\text { based on Site } 300 \\
\text { measured soil } \\
\text { concentrations } \\
\text { (p. B-55) }\end{array}$ & $\begin{array}{l}\text { S300EWTF } \\
\text { MammalRREI } \\
\text { S300Ranch } \\
\text { MammalRREI } \\
\text { S300OtherLoc } \\
\text { MammalRREI }\end{array}$ & $\begin{array}{l}\text { Cols. C, S, N, T } \\
\text { Cols. C, S, N, T (same as previous) } \\
\text { Cols. C, S, N, T and W (same as previous) }\end{array}$ \\
\hline $\begin{array}{l}\text { Table B-23: } \\
\text { ESSLLS-min and } \\
\text { EHQLS-min for Burrowing } \\
\text { Owl based on Site } 300 \\
\text { measured soil } \\
\text { concentrations } \\
\text { (p. B-55) }\end{array}$ & $\begin{array}{l}\text { S300EWTF } \\
\text { AVIAN_ReptileRREI } \\
\text { S300Ranch } \\
\text { AVIAN_ReptileRREI } \\
\text { S300OtherLoc } \\
\text { AVIAN_ReptileRREI }\end{array}$ & $\begin{array}{l}\text { Cols. C, M, H, N } \\
\text { Cols. C, M, H, N } \\
\text { Cols. C, M, H, N and Q }\end{array}$ \\
\hline
\end{tabular}




\section{References}

Gallegos G.M., J.I. Daniels, and A.M. Wegrecki (2007), Human Health and Ecological Risk Assessment for the Operation of the Explosives Waste Treatment Facility at Site 300 of the Lawrence Livermore National Laboratory; Volume 1: Report of Results, Lawrence Livermore National Laboratory, Livermore, CA, UCRL-TR-216940 Vol 1, Rev. 3. 